\title{
Impactos del programa Alianzas para la Innovación en empresas del sector TIC de Caldas
}

\author{
The Impacts of the Partnerships for Innovation Program in the ICT Business Sector \\ in Caldas
}

\author{
J. M. Toro-Galvis ;O. L. Ocampo-López ; A. M. Ovalle-Castiblanco iD; \\ M. L. Serna-López iD
}

\begin{abstract}
The Partnerships for Innovation Program is a COLCIENCIAS initiative supported by Confecámaras, and it is intended to generate an innovative culture and way of thinking as well as the skills to design and develop innovative projects. The Chamber of Commerce of Manizales, Caldas (CCMPC) has led this program in the Coffee Region. The impacts of the Partnerships for Innovation Program have been identified through applied research of a descriptive nature which was developed by the Autonomous University of Manizales and the CCMPC in different sectors of the department of Caldas. Through the analysis of the indicators, the program achievements, and the barriers and drivers of innovation, the aforementioned impacts were evaluated in the nine (9) companies of the ITC sector that participated in the program. The results show advances in the strategy of training, management, and promotion of innovation which translate in to the following concrete results: $9 \%$ of cost savings, $22 \%$ of increase in sales, and a new product and a new service introduced into the market by each company. It must be emphasized that these companies know why to innovate and where the company is heading with such innovation. They must advance on specific issues such as collaborative culture and organizational learning for innovation management.
\end{abstract}

Index Terms - Development indicators, Innovation, Information Technology, Management, Organizational Change.

Resumen- El programa Alianzas para la Innovación es una iniciativa de COLCIENCIAS con el apoyo de Confecámaras que busca generar mentalidad y cultura innovadora, así como habilidades para diseñar y desarrollar proyectos de innovación. La Cámara de Comercio de Manizales por Caldas (CCMPC) ha liderado este programa en EI Eje Cafetero.

Este manuscrito fue enviado el 25 de enero de 2019 y aceptado el 25 de agosto de 2019. Este trabajo fue apoyado en parte por de la Universidad Autónoma de Manizales y la Cámara de Comercio de Manizales por Caldas bajo el código de proyecto 494-074.

J. M. Toro-Galvis, docente del Departamento de Mecánica y Producción de la Universidad Autónoma de Manizales, Colombia (e-mail: juana.torog@autonoma.edu.co).

O. L. Ocampo-López, docente del Departamento de Mecánica y Producción de la Universidad Autónoma de Manizales, Colombia (e-mail: olocampo@autonoma.edu.co).

A. M. Ovalle-Castiblanco, docente del Departamento de Mecánica y Producción de la Universidad Autónoma de Manizales, Colombia (e-mail: movalle@autonoma.edu.co).

M. L. Serna-López, Líder Área de Gestión de Innovación de la Cámara de Comercio de Manizales por Caldas, Colombia (e-mail: mlserna@ccm.org.co).
Los impactos de Alianzas para la Innovación han sido identificados a través de una investigación aplicada, de tipo descriptivo, desarrollada por la Universidad Autónoma de Manizales y la CCMPC en diferentes sectores para el departamento de Caldas. En el sector TIC fueron evaluados, en nueve (9) empresas que participaron del programa, mediante el análisis de los indicadores y logros del programa; las barreras e impulsores para la innovación. Los resultados permiten identificar avances en la estrategia de formación, gestión y fomento a la innovación que se traducen en resultados concretos tales como: 9\% de ahorro en costos; $22 \%$ de incremento en las ventas; un nuevo producto y un nuevo servicio introducido en el mercado por empresa. Se destaca que las empresas evaluadas tienen claro la razón para innovar y saben hacia dónde se dirige la empresa con la innovación; estas empresas deben avanzar en temas específicos tales como la cultura colaborativa y el aprendizaje organizacional para la gestión de la innovación.

Palabras clave- Cambio organizacional, Gestión, Indicadores de desarrollo, Innovación, Tecnología de la información.

\section{INTRODUCCIÓN}

$\mathrm{E}$ N Colombia, los esfuerzos para impulsar la Ciencia, Tecnología e Innovación (CTI) están dirigidos a mejorar la productividad y competitividad [1], [2], [3], [4].

La CTI se constituye por tanto, en la columna vertebral para avanzar en innovación y lograr impactos empresariales que se traduzcan en beneficios para la sociedad [3], [5]. A nivel empresarial, se requiere avanzar en sofisticación e innovación, factores que afectan los índices nacionales y regionales de competitividad e innovación [6], [7]. De igual manera, es necesario mejorar el desarrollo del capital humano e infraestructura, así como la incorporación de estrategias, técnicas y metodologías de la gestión de la innovación en las organizaciones [1], [2].

En este sentido, se requiere consolidar sistemas de innovación que faciliten e incentiven el flujo de conocimiento entre los diversos actores para incorporar la CTI [8]. Es así como el gobierno nacional, a través de Colciencias y 
Confecámaras, ha implementado programas, como "Pactos por la Innovación", que buscan aumentar la inversión de las Actividades de Ciencia, Tecnología e Innovación (ACTI) al 1\% del PIB para el 2018.

El Eje Cafetero ha sido pionero en estos programas que fomentan la mentalidad y cultura innovadora, tal como Eje Innova y Pactos por la Innovación. Este último incorpora programas específicos como Alianzas para la innovación y Sistemas de Innovación, que son operados por la Cámara de Comercio de Manizales por Caldas (CCMPC) con el apoyo de otras Cámaras de Comercio de la región [9].

La CCMPC está realizando la evaluación de los impactos del programa Alianzas para la Innovación mediante un proyecto de investigación aplicada con el apoyo de investigadores de la Universidad Autónoma de Manizales (UAM). Esta evaluación considera los impactos del programa en mentalidad y cultura innovadora, la incorporación de la Ruta de la Innovación y los indicadores de innovación en empresas participantes. En este artículo se muestran los resultados para el sector de Tecnología de Información y Comunicación (TIC).

La conexión entre las TIC y la CTI es clave para lograr una sociedad basada en el conocimiento, donde las nuevas tecnologías contribuyan a la solución efectiva de los problemas del país [10]. Es por esto que el sector TIC es prioritario a nivel nacional y es considerado como la nueva "locomotora" para el desarrollo [11].

En Colombia, la cadena de valor de este sector comprende las siguientes dimensiones: 1) Infraestructura que soporta la utilización de servicios y productos; 2) Fabricación y/o venta de bienes TIC; 3) Producción de servicios TIC 4) Industrias de plataformas digitales 5) Investigación, desarrollo e innovación en TIC [12]. Es por esta complejidad y transversalidad que "el sector TIC no puede medirse como una sola actividad económica del PIB" [12].

A través del intercambio de información, se evidencia aumento en la demanda del sector y alianzas comerciales, que se traducen en una participación del 6\% en el PIB nacional [11]. El crecimiento económico anual de este sector, en el país, es mayor al de potencias mundiales -Reino Unido, Alemania, USA- y latinoamericanas -Brasil y Chile- [12].

Colombia cuenta con alrededor de cuatro mil empresas con actividades económicas en el sector TIC; se destaca la participación de las micro, medianas y pequeñas empresas (mipymes) y en especial de las microempresas que representan más del 50\% del sector [13].

En el departamento de Caldas, las empresas del sector TIC trabajan en áreas estratégicas e innovadoras como: Turismo, biotecnología, diseño de materiales, electrónica, salud, logística, energía, recursos naturales y aplicaciones civiles de desarrollo, entre otras [14]. No obstante, la cadena TIC está altamente especializada en desarrollo de software y aplicaciones para servicios empresariales [14].

En Manizales, capital del Departamento de Caldas, se identifican para el Sector TIC, brechas estratégicas, tecnológicas, de conocimiento, en soporte operativo, en financiación y de política pública. Entre las principales brechas se tiene una baja capacidad de I+D+I en la región, la necesidad de establecer procesos avanzados en I+D+I, la obsolescencia tecnológica y la falta de asistencia técnica para escalar procesos, productos y/o servicios [15].

El cierre de estas brechas que limitan la competitividad es uno de los objetivos trazados por las instituciones participantes del Ecosistema de Ciencia, Tecnología e Innovación en el Departamento de Caldas. La CCMPC y la UAM, actores claves del Ecosistema evaluaron los impactos del Programa Alianzas para la innovación en la capacidad de I+D+I en la región y específicamente en el sector TIC. A continuación, se presentan los resultados considerando los indicadores de innovación, beneficios, barreras e impulsores de la innovación y el avance en la incorporación de la Ruta de la Innovación.

\section{II.GESTIÓN DE LA INNOVACIÓN}

La innovación es uno de los pilares para la sostenibilidad económica, evolución y crecimiento de las organizaciones a través de la creación y la captura de valor [1], [16], [17]; permite mejorar el posicionamiento y la competitividad de una empresa mediante la introducción de nuevas y mejores ideas [18]. Implica mejoras en los procesos organizacionales que contribuyen tanto a la competitividad como a la productividad empresarial [18].

La innovación es un proceso sistemático que debe ser gestionado en las organizaciones considerando diferentes etapas que van desde la ideación, hasta la comercialización [19], [20], [21] pues solo se reconoce la innovación cuando es aceptada por el mercado [18]. Debido al avance de la globalización y la digitalización, las PYME deben enfrentar varios desafíos en su gestión de la innovación [22].

Para la innovación se requiere una cultura colaborativa en las organizaciones que conlleve al mejoramiento continuo de sus productos y procesos [23], [24], [25]; y a la resolución de problemas para generar cambios estructurales [20], [26].

Este mejoramiento continuo se da a través de la relación entre el aprendizaje y las capacidades de innovación; que consisten en transformar continuamente nuevos conocimientos e ideas en nuevos productos, procesos y sistemas [27]. estos aspectos son relevantes para la generación de innovaciones, a través de la experiencia y factores culturales de los individuos de la organización [28], [29], [30].

Fortalecer estas capacidades de innovación resulta importante para mantener la participación de toda la 
organización; las técnicas de creatividad, la exploración y aplicación de nuevos conocimientos con un equipo para la innovación, permiten la generación de valor y de ventajas competitivas [29], [31], [32].

Por otra parte, la exploración del entorno permite la identificación de amenazas y oportunidades, nuevos segmentos de mercados y necesidades del consumidor, que conlleven a la generación de innovaciones y faciliten la conformación de una red estratégica, la cual es clave para la gestión de la innovación al interior de la organización [33], [34], [35], [36].

Para una correcta gestión de la innovación se deben designar los recursos necesarios para la creación de conocimientos e ideas, estimular la creatividad e invertir tiempo en innovar [37], [38]. Para tal efecto, se debe estructurar un equipo de apoyo, elaborar un plan de acción, formular proyectos, y definir los indicadores de medición y evaluación de la innovación para garantizar un adecuado proceso, alineado a la estrategia organizacional [38], [39]. En este sentido, una correcta identificación de los impulsores, los beneficios y las barreras de la innovación permite un mejor análisis de las fortalezas y debilidades internas, de tal manera que se prioricen las inversiones en programas para desarrollar cultura, capacidades y equipos para la innovación [40].

El Gobierno Nacional apoya el fortalecimiento en innovación a través de programas específicos como Alianzas para la Innovación que busca fomentar mentalidad y cultura innovadora, incrementar las capacidades de innovación y el desarrollo de proyectos de innovación [41].

El programa Alianzas para la Innovación define el proceso de innovación como la aplicación de nuevos conocimiento y tecnologías, desarrollados al interior de la organización, con colaboraciones internas y externas, incluyendo todas las actividades científicas, tecnológicas organizativas, financieras y comerciales que conducen a la innovación, con el fin de generar soluciones de alto impacto y novedosas que tengan éxito en el mercado [42].

Para tal fin, se ha establecido una guía denominada "La Ruta de la Innovación" dirigida a los empresarios que facilita comprender las etapas para la incorporación de actividades de innovación y de estrategias de trasformación cultural, a partir de la generación de capacidades de innovación [40]. Dicha guía comprende las siguientes etapas:

A. Estrategia de Innovación: Una organización que desee generar innovaciones deben establecer principalmente el concepto de innovación; los focos, los recursos y los incentivos para innovar. La estrategia de innovación debe alinearse a la estrategia empresarial.

B. Detección de oportunidades: Las empresas deben crear mecanismos que les permitan entender y explorar el mercado, los clientes, la industria y las tecnologías, con el fin de identificar y estructurar los problemas para innovar.

C.Generación de ideas: Esta etapa se realiza de manera secuencial pues la generación de ideas surge una vez se hayan detectado las oportunidades, los focos y los problemas para la innovación. Es una de las etapas más importantes pues los participantes deben retarse para generar soluciones no convencionales que conlleven diferenciación y solución al reto propuesto.

D.Conceptualización de ideas: Esta etapa permite estructurar, complementar y detallar las ideas generadas en la etapa anterior; por tanto, las ideas crecen y se fortalecen para convertirse en un proyecto futuro de innovación.

E. Selección y priorización: Comprende la selección de las mejores ideas para asegurar que el proyecto incluya los recursos necesarios para la materialización de las ideas y el logro de las innovaciones.

F. Experimentación/prototipado temprano: En esta etapa se construye una base sólida para la idea priorizada; se valida la idea propuesta a través de prototipos u otros mecanismos, de tal manera que refleje las características, funcionalidades y beneficios a una escala mínima de realidad, de tal forma que se pueda ajustar el modelo.

G.Estructuración de proyectos: Comprende la formulación del proyecto de innovación, a partir de la idea validada. En esta etapa se establecen objetivos, metodología, resultados, presupuesto y cronograma, para el proyecto específico.

H.Ejecución de proyectos: En esta etapa se materializa la idea en el mercado y se realiza la medición de los resultados esperados para la empresa.

\section{Metodología}

Este estudio fue producto de una investigación explicativa, en el marco del programa Alianzas para la Innovación en Caldas. Para su desarrollo se empleó información cualitativa y cuantitativa que fue recopilada por técnicas de entrevista y grupo focal a las empresas seleccionadas.

La unidad de análisis fue la empresa. Como criterios de inclusión se consideraron: Empresas TIC de Caldas participantes del programa Alianzas para la Innovación e inscritas en la Cámara de Comercio de Manizales por Caldas (CCMPC). Fueron seleccionadas 14 empresas del sector TIC considerando los criterios de inclusión; de las cuales 9 aceptaron participar en la investigación. En la Tabla I, se listan las empresas objeto de análisis. 
TABLA I.

EMPRESAS PARTICIPANTES DEL ESTUDIO

\begin{tabular}{ll}
\hline \hline \multicolumn{1}{c}{ Nombre de la empresa } & \multicolumn{1}{c}{$\begin{array}{c}\text { Tamaño } \\
\text { organizacional }\end{array}$} \\
\hline ALSUS IT GROUP & Microempresa \\
ACID HONEY HOUSE & Microempresa \\
AUTOMATIZACIÓN Y VIRTUALIZACIÓN & Microempresa \\
INGENIERÍA SAS & Mediana \\
ALVERTO ÁLVAREZ LÓPEZ & Mediana \\
HEINSOHN BUSINESS TECNOLOGY & Pequeña \\
SIGMA INGENIERÍA SA & Microempresa \\
SKYNET DOMOTICS SAS & Microempresa \\
UNDER ZERO PRODUCCIONES Y EVENTOS & Pequeña \\
WIXFLIX &
\end{tabular}

\section{A. Descripción del instrumento de medición:}

El instrumento para la recolección de la información fue elaborado por el equipo de investigadores de la UAM y la CCMPC, considerando los lineamientos de la Ruta de la Innovación y los objetivos de la Estrategia Pactos por la Innovación. El instrumento fue validado mediante prueba piloto. Entre los componentes evaluados se tienen:

- General y percepción: Fueron analizados aspectos como el tamaño de la organización, las características de las empresas (familiares o no), el momento de vida, los conceptos de tecnología e innovación, la formación del equipo que apoya el proceso de $\mathrm{I}+\mathrm{D}+\mathrm{i}$, la participación en programas regionales o nacionales que promueven la innovación, y la percepción del programa alianzas para la innovación.

- Trazabilidad: Se evaluaron los impactos del programa Alianzas para la Innovación desde dos perspectivas, vivencia personal y organizacional; se analizaron los impactos específicos mediante indicadores clave como: número de nuevos productos, servicios, mercados, empleos, porcentaje de ventas y ahorros en costos.

- Impulsores y barreras: Fueron analizados los aspectos que favorecían y limitaban los procesos de innovación en la organización.

Una vez recolectada la información, se consolidó y realizó el análisis de estadística descriptiva, mediante análisis gráficos e histogramas de frecuencias, con el software Microsoft Excel; se incluyeron categorías de clasificación mediante código de colores que facilita la devolución de los resultados a la empresa.

\section{Resultados}

La totalidad de las empresas evaluadas destaca la importancia de la innovación para la gestión empresarial. Es así como los encuestados concuerdan con la afirmación que la gestión de la Innovación contribuye al desarrollo empresarial fortaleciendo las capacidades tecnológicas e incrementando la productividad, y competitividad [43].

Sin embargo, sólo el $44 \%$ de las empresas evaluadas ha incorporado un proceso de Gestión de la Innovación. Los empresarios afirman que la continuidad a los programas específicos para el entrenamiento en innovación y el aprendizaje de técnicas de gestión de la innovación, es fundamental en el sector TIC, en especial por la alta proporción de mipymes. Por otra parte, recalcan la importancia de establecer estrategias de innovación, y avanzar en la implementación del proceso de innovación, considerando las características y limitaciones particulares.

El equipo que apoya los procesos de Investigación, Desarrollo e Innovación (I+D+i) es heterogéneo, conformado por profesiones con nivel de posgrado y pregrado, pero también por técnicos, tecnólogos y bachilleres que ha sido capacitados en estos temas. Cabe resaltar que un equipo ideal de I+D+i es conformado por personas con diversas profesiones, en diferentes niveles [31], de tal manera que se puedan generar ideas más novedosas y diversas. Para el sector TIC, es preferible un equipo de $\mathrm{I}+\mathrm{D}+\mathrm{i}$ a nivel multidisciplinar y considerar además la consultoría en I+D+i dadas las diferentes aplicaciones y ámbitos de trabajo.

\section{A. Ruta de la Innovación}

Las empresas analizadas consideran aspectos clave desde el punto de vista estratégico como: la razón para innovar (100\% de las empresas evaluadas), el foco de la innovación organizacional (78\%), el futuro de la compañía con la innovación (89\%), tal como se ilustra Fig 1. La innovación se potencializa cuando es incorporada en la estrategia organizacional [40]. Las herramientas de mayor aplicación para la definición del componente estratégico de innovación son Vigilancia Tecnológica y DOFA. Por otra parte, la mayoría de los encuestados (78\%) reconocen la contribución del Programa Alianzas para la Innovación para facilitar la definición de la estrategia de innovación; no obstante, el 56\% manifiesta tener debilidades en la creación de incentivos para innovar. 


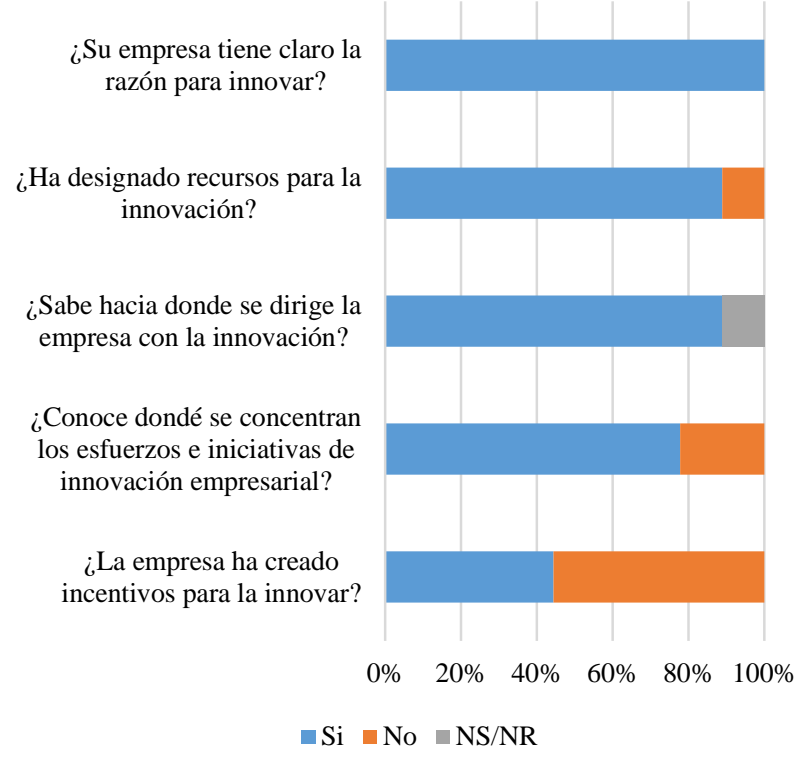

Fig 1. Estrategia de innovación

La totalidad de las empresas aplica técnicas para la detección de oportunidades a través de la exploración de su entorno convirtiéndolo en un reto a sus capacidades de innovación (Fig 2). Entre las herramientas empleadas por las empresas para la detección de oportunidades se tienen: Entrevistas, Investigación de mercados, análisis de tendencias, Problem Solving y misiones de observación.

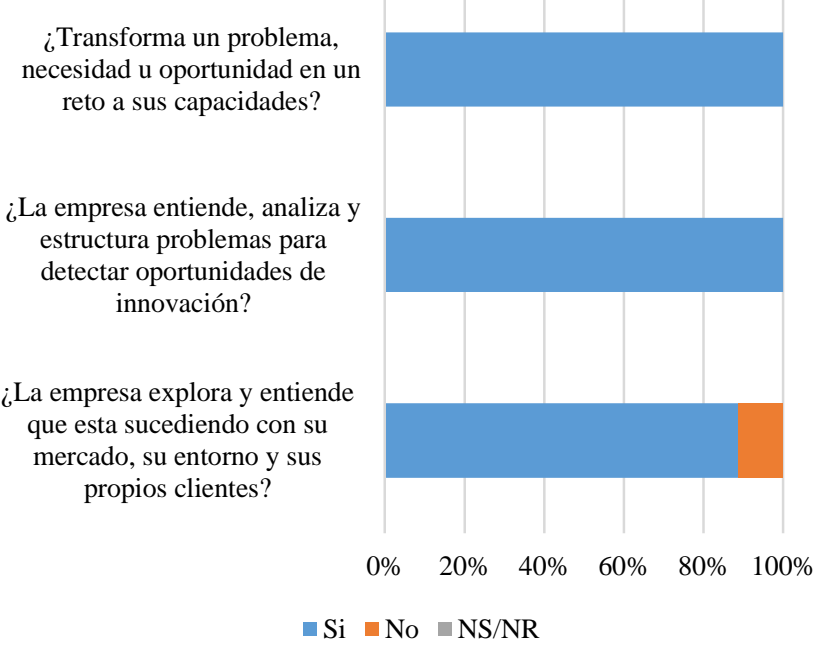

Fig 2. Detección de oportunidades para innovar

El $100 \%$ de las empresas analizadas ha identificado, según los hallazgos de la exploración del entorno, nuevas oportunidades para innovar y el $89 \%$ ha identificado problemas que lo inspiren a realizar innovaciones (Fig 3). Las herramientas empleadas para esta etapa de la Ruta de la Innovación son similares a las utilizadas en detección de oportunidades. Para este sector, la identificación de problemas, desafíos y mercados son necesarios para el funcionamiento de la innovación [40] y es transversal a los demás sectores de la economía.

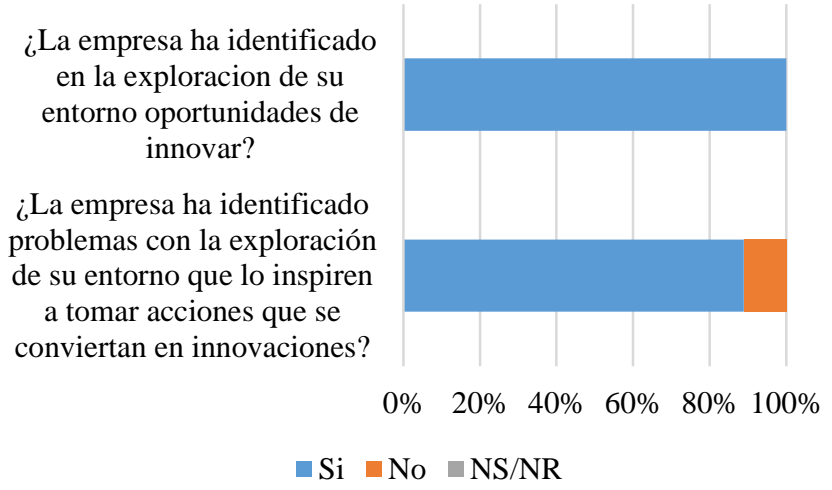

Fig 3. Hallazgos y descubrimientos en el entorno

El 89\% de las empresas participantes en el estudio establece criterios para la selección de ideas; un $78 \%$ realiza sesiones de creatividad para generar ideas de mayor impacto en su medio (Fig 4); estas etapas son fuente de inspiración y reto para las organizaciones, pues permiten salir de soluciones convencionales mediante la creación de ideas novedosas y diferentes [40]. Las herramientas de mayor uso para generar, conceptualizar y fortalecer las ideas en las empresas del sector TIC fueron Lluvia de ideas, Canvas Business Model Generation y Cocreación.

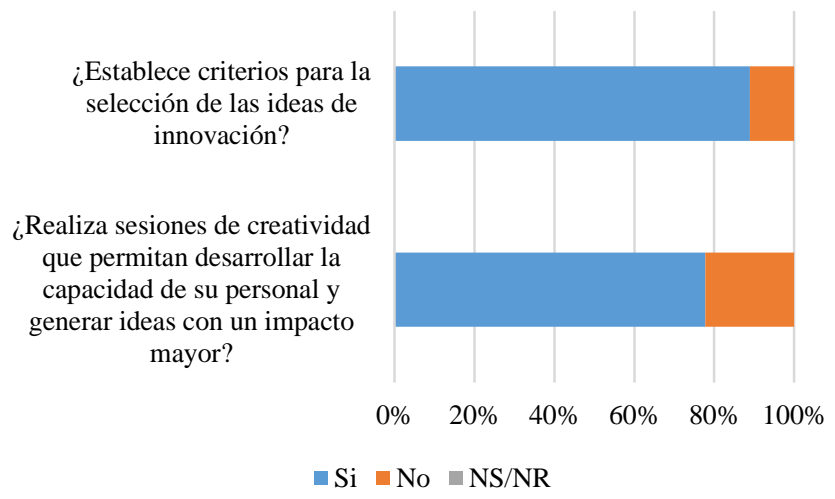

Fig 4. Generación, conceptualización y fortalecimiento de ideas

La mayoría de las empresas analizadas tienen un plan de validación de ideas (89\%), una ruta adecuada para materializar la idea (89\%) y validan esas ideas a través de prototipos (78\%) (Fig 5). Las herramientas de mayor uso son los dibujos o esquemas, los modelos o prototipos físicos, la presentación o discurso y el mapa conceptual. Es necesario recalcar que la mejor estrategia para experimentar, validar, ajustar y fortalecer una idea es la creación de prototipos tempranos que permitan fallar rápido y evitar inversiones innecesarias, antes de que la idea se materialice en un proyecto de innovación [40]. 


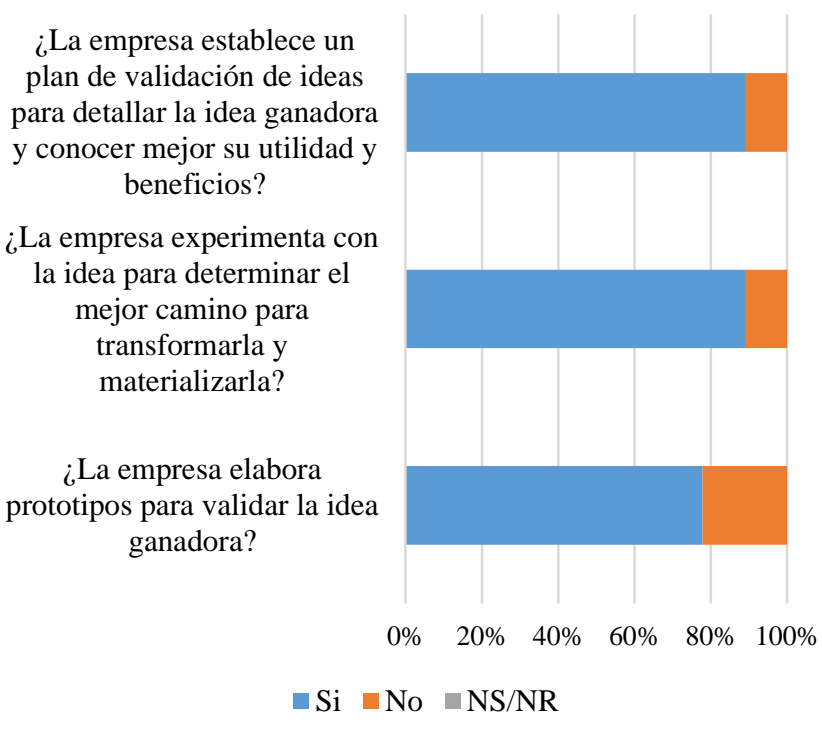

Fig 5. Experimentación y validación de ideas

El $67 \%$ de la empresas TIC analizadas ha formulado proyectos de innovación y el $89 \%$ asegura que los proyectos formulados se ejecutan y se realiza seguimiento (Fig 6); sin embargo, se evidencia que la mayoría de las empresas aún no tiene establecido Portafolios de proyectos de innovación, los cuales deben considerar el cumplimiento de los objetivos estratégicos de innovación definidos por la organización [40]. Las herramientas más empleadas para la formulación y ejecución de proyecto fueron Business Model Canvas y Lecciones Aprendidas.

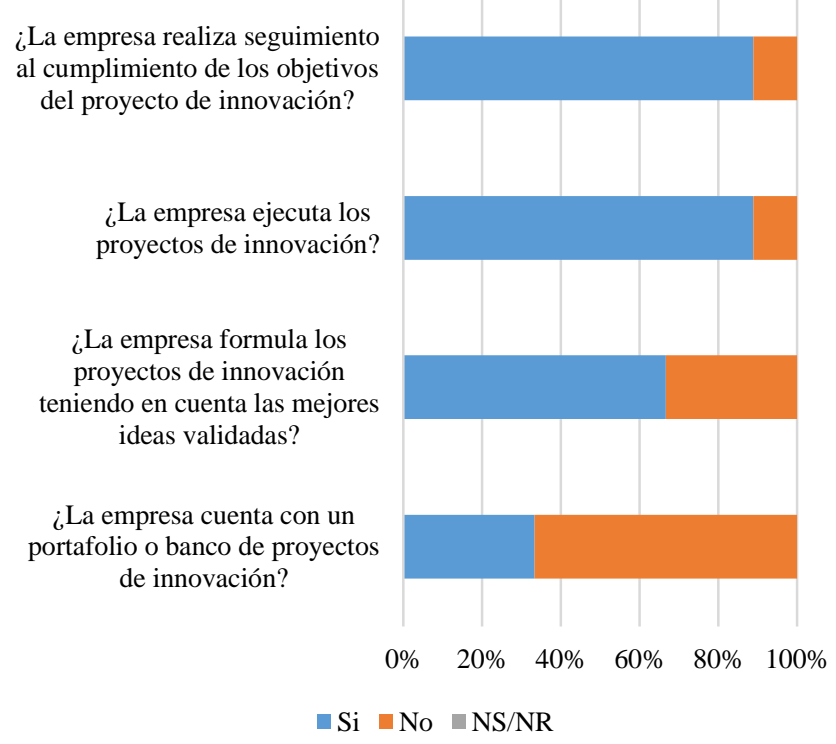

Fig 6. Formulación y ejecución de proyectos

\section{B. Impactos del programa Alianzas para la Innovación}

Los impactos fueron analizados desde la percepción a nivel organizacional (Fig 7) y personal (Fig 8). A continuación, se describen los principales impactos, mostrando entre paréntesis el porcentaje de empresas que calificaron Muy Alto y Alto.

A nivel organizacional, la consolidación de un líder o equipo gestor de innovación (67\%), los beneficios obtenidos de los proyectos de innovación (22\%), la ejecución de proyectos de innovación (44\%) y una cultura de innovación (44\%) son los impactos más importantes que perciben los entrevistados del programa Alianzas para la Innovación al interior de las organizaciones (Fig 7). De esta manera, se cumple con los objetivos del programa que son fomentar la innovación y las capacidades en la organización, pues para mantener la cultura de innovación se requiere un equipo que genera ideas y formule proyectos [38].

Si bien algunas empresas avanzaron en etapas como el establecimiento del proceso de innovación y la construcción del portafolio de innovación, son temas que no se profundizan en Alianzas para la innovación, pues hacen parte de programas posteriores como Sistemas para la Innovación.

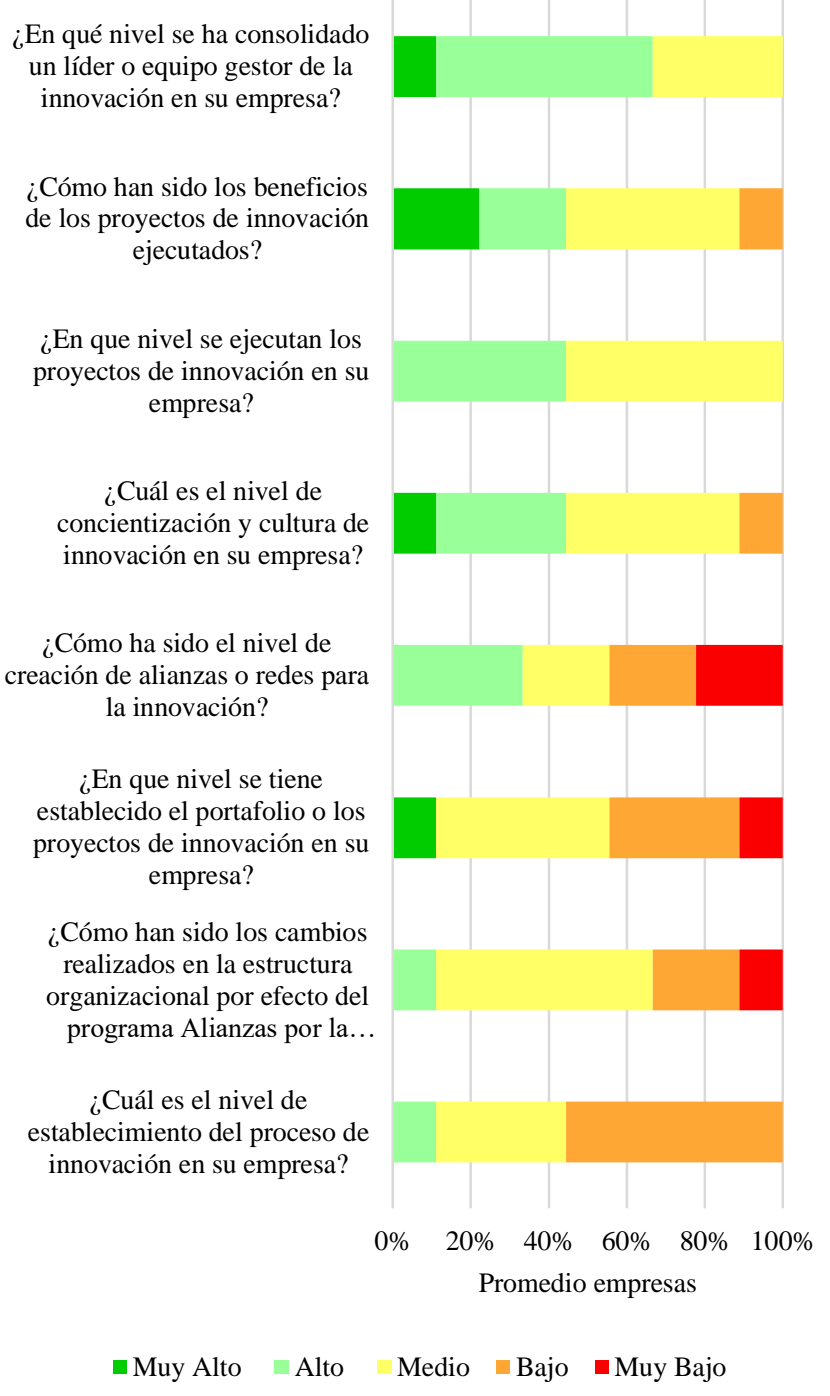

Fig 7. Impacto organizacional del programa Alianzas para la Innovación 
A nivel personal, los encuestados refieren que el mayor impacto del programa Alianzas para la Innovación fue la generación de una cultura de innovación (89\%) y una mentalidad innovadora (66\%), cumpliendo así con los objetivos trazados en este programa; no obstante, se requiere avanzar en el conocimiento de la Ruta de Innovación (Fig 8). La creación de una mentalidad y cultura de innovación genera motivación y fomenta el aprendizaje, trabajo colaborativo y el aporte de nuevas ideas [44].

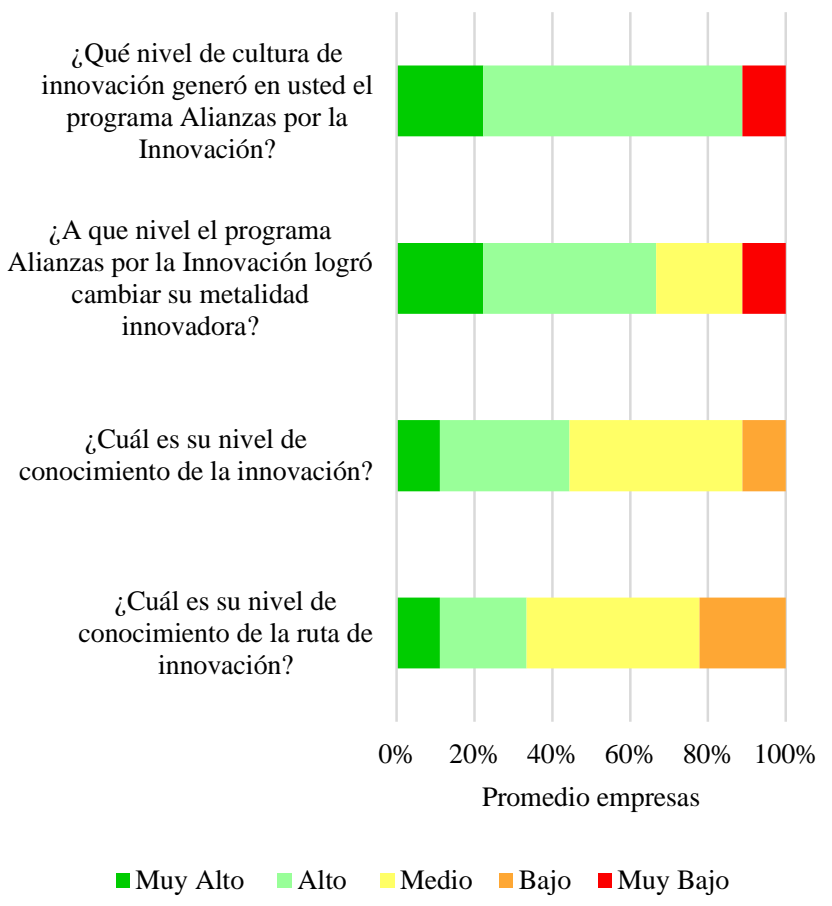

Fig 8. Impacto personal del programa Alianzas para la Innovación

\section{Indicadores de Innovación}

Por otra parte, los impactos del programa Alianzas para la Innovación fueron evaluados mediante indicadores de Innovación que se presentan en la Tabla II.

TABLA II.

INDICADORES DE IMPACTO DEL PROGRAMA ALIANZAS PARA LA INNOVACIÓN

\begin{tabular}{lcc}
\hline \hline \multicolumn{1}{c}{ Indicador } & Promedio & Máximo \\
\hline Número de nuevos empleos generados & 1 & 2 \\
Número de productos mejorados & 1 & 2 \\
Número de servicios mejorados & 1 & 2 \\
$\begin{array}{l}\text { Número de nuevos productos introducidos } \\
\text { al mercado }\end{array}$ & 1 & 3 \\
$\begin{array}{l}\text { Número de nuevos servicios introducidos al } \\
\text { mercado }\end{array}$ & 1 & 3 \\
Número de nuevos mercados & 0 & 1 \\
Porcentaje de ahorro en costos & $9 \%$ & $30 \%$ \\
Porcentaje de aumento en las ventas & $22 \%$ & $100 \%$ \\
\hline \hline
\end{tabular}

Entre los principales impactos del Programa se destaca el aumento en las ventas y los ahorros en costos. Se evaluaron además impactos posteriores considerando los indicadores cítricos de innovación (Tabla III). Se refleja un mayor dinamismo en temas de innovación, considerando los niveles de inversión, las ideas generadas y los productos y servicios introducidos en el último año.

TABLA III INDICADORES CRÍTICOS DE INNOVACIÓN

\begin{tabular}{lcc}
\hline \hline \multicolumn{1}{c}{ Indicador } & Promedio & Máximo \\
\hline $\begin{array}{l}\text { Número de ideas evaluadas de productos o } \\
\text { servicios en el último año }\end{array}$ & 4 & 12 \\
$\begin{array}{l}\text { Número de productos o servicios } \\
\text { introducidos en el último año } \\
\begin{array}{l}\text { Tiempo promedio de la generación de la } \\
\text { idea hasta la introducción al mercado } \\
\text { (meses) }\end{array}\end{array}$ & 2 & 6 \\
$\begin{array}{l}\text { Porcentaje de las ventas de los productos/ } \\
\text { servicios nuevos o mejorados en los últimos } \\
\text { tres años }\end{array}$ & $18 \%$ & $80 \%$ \\
$\begin{array}{l}\text { Inversión en Innovación en el último año } \\
\text { como porcentaje de las ventas }\end{array}$ & $4 \%$ & $20 \%$ \\
\hline \hline
\end{tabular}

\section{Beneficios, Impulsores y barreras para la innovación}

La Fig 9 presenta los beneficios de la innovación en las empresas evaluadas del sector TIC.

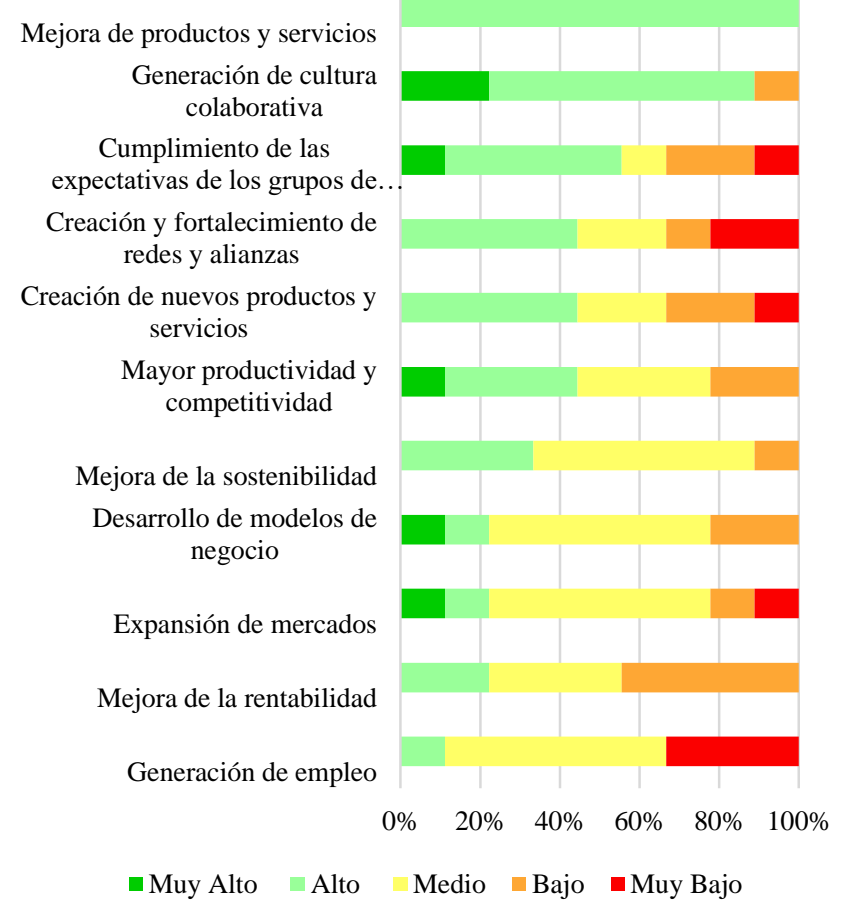

Fig 9. Beneficios de la Innovación en la Organización

Considerando las categorías alto y muy alto, la mejora de productos y servicios $(100 \%)$, la generación de una cultura colaborativa (89\%) y el cumplimiento de las expectativas de los grupos de interés (55\%), son los principales beneficios. Por otra parte, no se perciben como beneficios importantes la generación de empleo, la mejora de la rentabilidad y la expansión de mercados. En este sector la creación y captura de valor [16] se 
traduce en la mejora de nuevos productos o servicios, para lo cual la generación de ideas o nuevos enfoques, mediante una cultura colaborativa, facilita el proceso de innovación [23].

Por otra parte, la Fig 10 muestra los impulsores de la innovación en las empresas del Sector TIC analizadas. Para los empresarios del sector TIC evaluados, la innovación contribuye a la mejora de la productividad y la competitividad, que son resultados esperados de las estrategias de innovación [1], [2]. Es así como entre los principales impulsores referidos por los encuestados figura el posicionamiento de bienes (89\%), la generación de ventajas competitivas (89\%), el cumplimiento de metas $(77 \%)$ y la mejora de la productividad $(66 \%)$. No obstante, no se perciben como impulsores relevantes la reducción de costos, los requerimientos de grupos de interés y las estrategias de responsabilidad social.

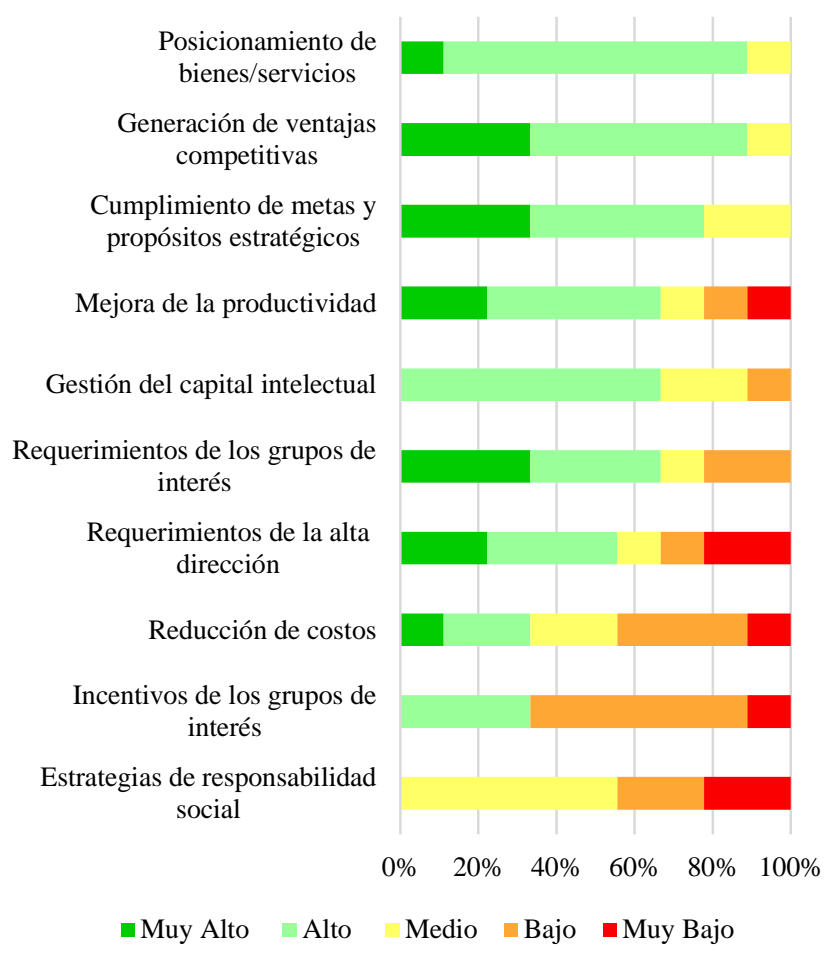

Fig 10. Impulsores de la Innovación

Finalmente, se presentan las barreras para la innovación que perciben los empresarios del sector TIC (Fig 11). Considerando las categorías muy alta y alta, la falta de tiempo (78\%) y el presupuesto y financiamiento (66\%) son las principales barreras que han experimentado durante el trabajo en innovación. Es importante anotar, que no se perciben como barreras el compromiso de la gerencia, la competencia del personal y la cultura organizacional, lo cual es un reflejo del avance en la cultura y capacidades de innovación en la organización.

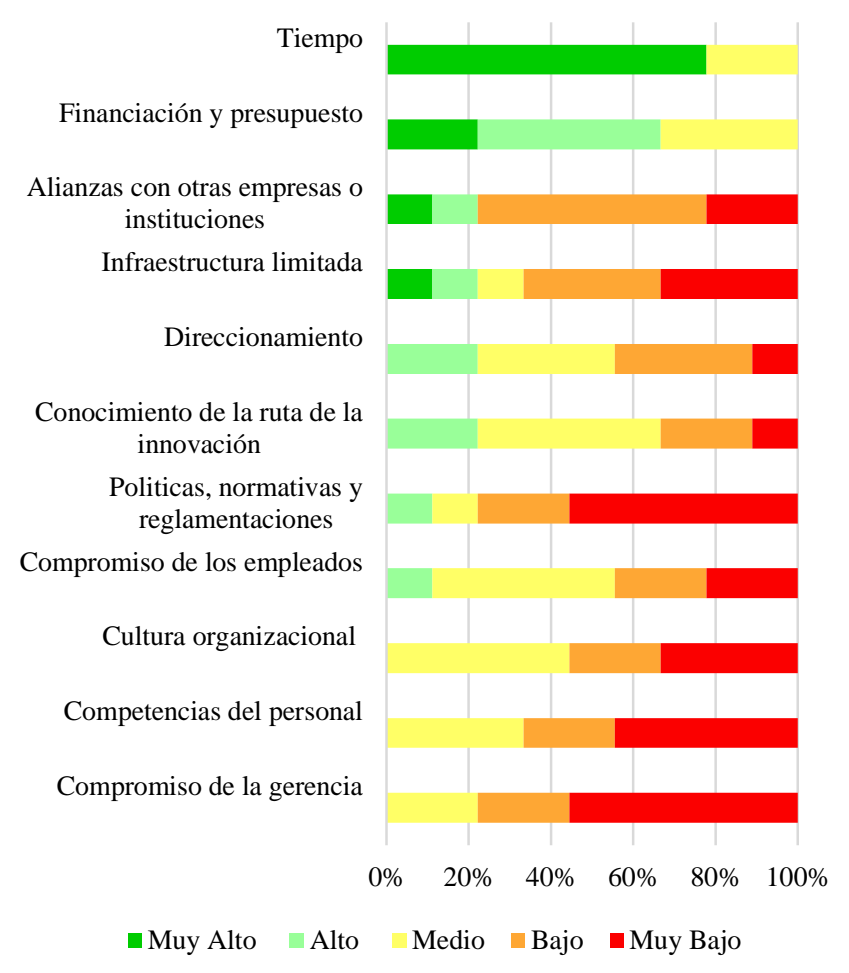

Fig 11. Barreras de la Innovación

La correcta Gestión de la Innovación implica una transición desde el aprendizaje a la práctica, para lo cual se requiere un trabajo inicial de fortalecimiento de competencias en innovación [45], en mentalidad y cultura innovadora que son los aprendizajes del programa Alianzas para la Innovación.

\section{CONCLUSIONES}

La evaluación de los impactos del programa Alianzas para la Innovación, en empresas del sector TIC en el departamento de Caldas, permite concluir que se logra el propósito principal que es la generación una mentalidad y cultura innovadora en las organizaciones participantes. Se evidencia la mejora de las capacidades de innovación mediante la consolidación de un equipo gestor o líder de innovación a nivel organizacional. Sin embargo, se requiere fortalecer la gestión de la innovación en las empresas, pues más de la mitad de las empresas evaluadas no cuentan con un proceso de gestión de la innovación establecido.

Los impactos del programa Alianzas para la Innovación en las empresas del sector TIC del departamento de Caldas se traducen en indicadores específicos de innovación entre los que se destaca: el incremento en las ventas ( $22 \%$ promedio, $100 \%$ máximo), el ahorro en costos ( $9 \%$ promedio, $30 \%$ máximo), por efecto de la introducción de nuevos productos y servicios (1 promedio, 3 máximo) y la mejora de productos y servicios (1 promedio, 2 máximo). Se evidencia además un mayor dinamismo en indicadores críticos de innovación, que se 
reflejan en las ventas de los nuevos productos y servicios (18\% promedio, $80 \%$ máximo). Sin embargo, no se perciben impactos importantes en las empresas evaluadas, en este sector, en la generación de empleo.

Las empresas del sector TIC del departamento de Caldas participantes del Programa Alianzas para la Innovación demuestran avances en las siguientes etapas de la Ruta de la Innovación: 1. Estrategia de Innovación, 2. Detección de oportunidades, 3. Hallazgos y descubrimientos en el entorno, 4. Generación, conceptualización y fortalecimiento de ideas, 5. Experimentación y validación de ideas, 6. Formulación y Ejecución de Proyectos. Sin embargo, requiere una mayor comprensión de la Ruta de la Innovación, en especial de las actividades para la formulación y ejecución de proyectos de innovación.

La mejora de productos y servicios, la generación de la cultura colaborativa y el cumplimiento de expectativas de los grupos de interés son los principales beneficios de la innovación que perciben los empresarios del sector TIC del departamento de Caldas que participaron del Programa Alianzas para la innovación. Por otra parte, estos empresarios tienen como principales impulsores de la innovación, el posicionamiento de productos y servicios, la generación de ventajas competitivas, el cumplimiento de propósitos estratégicos y el mejoramiento de la productividad. Finalmente, el tiempo, la financiación y el presupuesto son las principales barreras para la innovación que perciben los empresarios evaluados en este sector.

\section{RECOMENDACIONES}

VII.

Los empresarios del sector TIC recomiendan que el programa se oferte de manera sectorizada para que las técnicas empleadas sean de mayor pertinencia y faciliten el aprendizaje organizacional. Solicitan un mayor apoyo en el seguimiento de los proyectos de innovación.

Las empresas del sector TIC deben continuar fortaleciendo sus capacidades en innovación, su mentalidad y cultura innovadora y avanzar en programas específicos como Sistemas de Innovación que les permite la creación y consolidación de la gestión de la innovación.

La generación de redes y alianzas no sólo facilita gestionar el conocimiento, estimular la creatividad y el aprendizaje organizacional sino también fortalecer las capacidades de innovación. Por tanto, se requiere el apoyo Universidad Empresa - Estado y el fomento a estos programas clave como son Alianzas para la innovación.

\section{REFERENCIAS}

J. C. Naranjo Valencia and G. Calderón Hernández, "La Investigación en Innovación en Colombia y México. Un análisis desde la difusión en revistas científicas," Dyna, vol. 77, no. 162, pp.
191-203, 2010

[2] OCDE, Estudios de la OCDE de politicas de innovación: Colombia. Paris: OECD Publishing, 2014. https://doi.org/10.1787/9789264204638-en.

[3] DPN, "Política Nacional De Desarrollo Productivo," 2016.

[4] Colciencias, Libro Verde 2030 Política Nacional de Ciencia e Innovación para el desarrollo sostenibles, María Isab. Bogotá, D.C, 2018.

[5] CPC, "Informe nacional de competitividad 2017-2018," 2017. https://doi.org/ISSN 2016-1430.

[6] DNP and OCyT, "Índice Departamental de Innovación para Colombia, 2017,” Bogotá, D.C, 2017.

[7] CPC, Índice Departamental de Competitividad. Bogotá, D.C: Universidad del Rosario, 2017.

[8] DPN, "Política Nacional de Ciencia, Tecnología e Innovación 20152025," Bogotá, D.C, Borrador 1, 2015.

[9] DPN, "Bases del Plan Nacional de Desarrollo 2014-2018," 2014. https://doi.org/10.1017/CBO9781107415324.004.

[10] Portafolio, "El rol de las TIC en la ciencia y la tecnología," 2017. [Online]. Available: https://www.portafolio.co/economia/el-rol-delas-tic-en-la-ciencia-y-la-tecnologia-510409. [Accessed: 09-Dec2018].

[11] La República, "Colombia se destaca con crecimiento de las TIC," 2017. [Online]. Available: https://www.larepublica.co/interneteconomy/colombia-se-destaca-con-crecimiento-de-las-tic-2464511. [Accessed: 09-Dec-2018].

[12] D. L. Sánchez et al., "Panorama TIC 2015," 2015.

[13] Colciencias, Plan Nacional de Ciencia Tecnología e Innovación para el desarrollo del sector de las Tecnologías de la Información y las Comunicaciones TIC 2017-2022. Bogotá, D.C, 2016.

[14] M. L. García Naranjo, M. Cardona Acevedo, and Y. Espinosa Pérez, "Estudio del comportamiento del sector de las TIC en la ciudad de Manizales 2012-2014," Manizales, 2014.

[15] Alcaldía de Manizales, "Agenda de Competitividad de Manizales," Manizales, 2014.

[16] GIMI, "Gestión de la Innovación conjunto de conocimientos guía sobre innovaciones disruptivas," Global Innovation Managment Institute, Cambridge, 2013.

[17] P. V. Robayo Acuña, "La innovación como proceso y su gestión en la organización: una aplicación para el sector gráfico colombiano," Suma Negocios, vol. 7, no. 16, pp. 125-140, 2016. https://doi.org/10.1016/j.sumneg.2016.02.007.

[18] OECD y Eurostat, Manual de Oslo, 3a Edición. 2005. https://doi.org/10.1787/9789264065659-es.

[19] R. Sancho, "Directrices de la OCDE para la obtención de indicadores de Ciencia y Tecnología," Madrid, 2001.

[20] C. L. Correa, V. Yepes, and E. Pellicer, "Factores determinantes y propuestas para la gestion de la innovacion en las empresas constructoras," Rev. Ing. Constr., vol. 22, no. 1, pp. 5-14, 2007. https://doi.org/10.4067/S0718-50732007000100001.

[21] F. Gault, "Defining and measuring innovation in all sectors of the economy," Res. Policy, vol. 47, no. 3, pp. 617-622, Apr. 2018. https://doi.org/10.1016/j.respol.2018.01.007.

[22] N. Niewöhner, L. Asmar, F. Wortmann, D. Röltgen, A. Kühn, and R. Dumitrescu, "29th CIRP Design 2019 (CIRP Design 2019). Design fields of agile innovation management in small and medium sized enterprises," Procedia CIRP, vol. 84, pp. 826-831, 2019. https://doi.org/10.1016/j.procir.2019.04.295.

[23] C. Zurbriggen and M. G. Lago, "Innovación y co-creación: Nuevos desafíos para las políticas públicas," Rev. Gestión Pública, vol. 3, no. 2, pp. 329-361, 2014.

[24] I. Zayas Barreras, D. Parra Acosta, R. López Arciniega, and J. de D. Torres Sánchez, "La innovación, competitividad y desarrollo tecnológico en las MIP y ME's del municipio de Angostura, Sinaloa," Rev. Mex. Ciencias Agrícolas, vol. 6, no. 3, pp. 603-617, 2015.

[25] C. K. Padilha and G. Gomes, "Innovation culture and performance in innovation of products and processes: a study in companies of textile industry," RAI Rev. Adm. e Inovação, vol. 13, no. 4, pp. 285-294, 2016. https://doi.org/10.1016/j.rai.2016.09.004.

[26] J. Švarc, J. Lažnjak, and M. Dabić, "Regional innovation culture in innovation laggard: A case of Croatia," Technol. Soc., vol. 58, pp. 101-123, Aug. 2019. https://doi.org/10.1016/J.TECHSOC.2019.03.006.

[27] O. S. I. Fayomi, I. P. Okokpujie, and G. U. Fayomi, "2nd International Conference on Sustainable Materials Processing and Manufacturing 
(SMPM 2019). An Innovation Concept towards Bridging the Gaps Between Teaching and Research," Procedia Manuf., vol. 35, pp. 775781, 2019. https://doi.org/10.1016/j.promfg.2019.06.022.

[28] J. Ossa, M. Prada, and F. Zapata, "La gestión del diseño en las empresas y su relación con las capacidades de innovación,” Medellín, 2013.

[29] V. García, M. Romerosa, and F. Lloréns, "Liderazgo transformacional: Influencia en la visión compartida, aprendizaje, innovación y resultado organizativo," Rev. Eur. Dir. y Econ. la Empres., vol. 16, no. 4, pp. 25-46, 2007.

[30] C. López, "Metodología para la evaluación de la organización informal en la perspectiva de las capacidades de innovación," Universidad Nacional de Coolombia, 2016.

[31] V. González Romá, "La innnovación en los equipos de trabajo," Papeles del Psicol., vol. 29, no. 1, pp. 32-40, 2008.

[32] A. Hidalgo and J. Albors, "8th International Conference on Frailty and Sarcopenia Research (ICFSR), March 1-3, 2018, Miami, USA, Symposia, Conference, Oral communications.," J. frailty aging, vol. 7, no. 1, pp. 61-91, 2018. https://doi.org/10.14283/jfa.2018.3.

[33] E. Conejero and J. Redondo, "Gestión y Análisis de Políticas Públicas," Gestión y Análisis Políticas Públicas, no. 15, pp. 1-21, 2016.

[34] M. E. Morales, C. Ortiz, Y. V. Duque, and P. A. Plata, "Estrategias para fortalecer capacidades de innovación: una visión desde micro y pequeñas empresas," Ciencia, docencia y Tecnol., vol. 27, no. 53, pp. 205-233, 2016.

[35] D. Jiménez and R. Sanz, "Innovación, aprendizaje organizativo y resultados empresariales. Un estudio empírico," Cuad. Econ. y Dir. la Empres., no. 29, pp. 31-56, 2006.

[36] J. G. Mendoza and A. Valenzuela, "Aprendizaje, innovación y gestión tecnológica en la pequeña empresa Un estudio de las industrias metalmecánica y de tecnologías de información en Sonora," Contaduría y Adm., vol. 59, no. 4, pp. 253-284, 2014.

[37] Observatorio Virtual de Trasferencia de Tecnología, "Guía práctica de transferencia de tecnología," 2015. [Online]. Available: http://www.ovtt.org/gestion_gestion_de_la_innovacion. [Accessed: 29-Mar-2018].

[38] J. G. Candía, L. García, C. L. Caro, and N. Romero, "Estrategia y cultura de innovación, gestión de los recursos y generación de ideas : prácticas para gestionar la innovación en empresas," Pensam. Y Gestión, vol. 36, pp. 107-133, 2014. https://doi.org/10.14482/pege.36.5567.

[39] Asociación de la Industria Navarra, "Guía práctica: La gestión de la Innovación en 8 pasos," Anain - Agencia Navarra De Innovación. Pamplona, p. 104, 2008. https://doi.org/NA-3710-2008.

[40] Confecámaras, Guía Ruta de la Innovación. 2016.

[41] Confecámaras and Colciencias, "Alianzas para la Innovación IV," ¿Qué es Alianzas para la Innovación?, 2018. [Online]. Available: http://alianzasparalainnovacion.co/. [Accessed: 21-Jan-2019].

[42] Colciencias, "Pactos por la Innovación," Autodiagnostico de Innovación, 2016. [Online]. Available: http://pactosporlainnovacion.colciencias.gov.co/privilegios-2/. [Accessed: 01-Apr-2018].

[43] AENOR, "Gestión de la I+D+i," 2013. [Online]. Available: https://www.aenor.es/aenor/certificacion/innovacion/innovacion_sis temas_166002.asp\#.WqWg-uhubIU. [Accessed: 11-Mar-2018].

[44] P. Ayala Espinoza, "Organizational Culture and Innovation.," Gestión las Pers. y Tecnol., no. 19, pp. 27-33, 2014.

[45] J. Freire and K. S. Brunet, "Políticas y prácticas para la construcción de una Universidad Digital," La Cuestión Univ., vol. 6, pp. 85-94, 2010 .

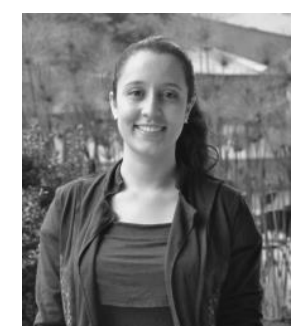

Juana María Toro, recibió el título de Ingeniera Industrial en 2016 y actualmente realiza una Maestría en Ingeniería en la Universidad Autónoma de Manizales-UAM.De 2017 a 2018 se vinculó como Joven Investigadora del grupo de Investigación de Diseño Mecánico y Desarrollo Industrial "Archytas" UAM. Desde 2018, ha sido Docente del
Departamento de Mecánica y Producción, y Líder de Semilleros de Investigación en la Unidad de Investigación de la UAM.

ORCID. http://orcid.org/0000-0002-8173-7906

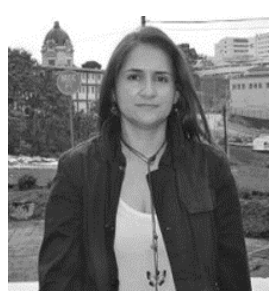

Olga Lucía Ocampo López, en 1994 obtuvo el título como Ingeniera Química; en 2012 culminó la Maestría en Ingeniería Química y en 2018 se tituló como Doctora en Ingeniería Automática de la Universidad Nacional de Colombia Sede Manizales. Es par evaluador reconocido por Colciencias en la categoría de Investigador Asociado. Desde el 2010, ha sido Docente del Departamento de Mecánica y Producción en la Universidad Autónoma de Manizales.

ORCID: http://orcid.org/0000-0002-6394-977X

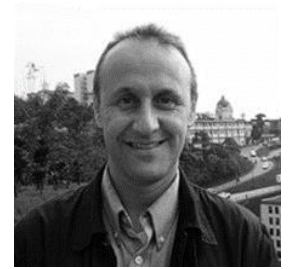

Alex Mauricio Ovalle Castiblanco, en 1994 obtuvo el título de Ingeniero Industrial en la Universidad Nacional de Colombia Sede Manizales, en 2013 finalizó una Maestría en Creatividad e Innovación en las Organizaciones y actualmente se encuentra realizando sus estudios de Doctorado en Ingeniería - Industria y Organizaciones. Es par evaluador reconocido por Colciencias en la categoría de Investigador Asociado. Desde 2003 es Docente del Departamento de Mecánica y Producción en la Universidad Autónoma de Manizales.

ORCID: http://orcid.org/0000-0002-1634-9456 